\title{
Sex differences in pharmacological treatment of major depressive disorder: results from the AMSP pharmacovigilance program from 2001 to 2017
}

\author{
Johanna Seifert ${ }^{1}$ (1) $\cdot$ Fabienne Führmann ${ }^{2} \cdot$ Matthias A. Reinhard $^{3} \cdot$ Rolf R. Engel $^{3} \cdot$ Xueqiong Bernegger $^{3,7}$. \\ Stefan Bleich ${ }^{1} \cdot$ Susanne Stübner ${ }^{3,4} \cdot$ Eckart Rüther $^{3,6} \cdot$ Sermin Toto $^{1} \cdot$ Renate Grohmann $^{3} \cdot$ Marcel Sieberer $^{5,7}$. \\ Waldemar Greil ${ }^{3,8}$
}

Received: 9 March 2021 / Accepted: 2 May 2021 / Published online: 11 May 2021

(c) The Author(s) 2021

\begin{abstract}
Data on drug prescription for outpatients with major depressive disorder (MDD) suggest women are more likely to be treated with psychotropic drugs, while data on sex differences regarding pharmacological treatment of psychiatric inpatients are currently not available. Drug utilization data from the program "Drug Safety in Psychiatry" (German: Arzneimittelsicherheit in der Psychiatrie, AMSP) of 44,418 psychiatric inpatients with MDD were analyzed for sex differences between 2001 and 2017. Sex differences were analyzed using relative risks (RR) and 95\% confidence intervals (95\% CI). Time trends were analyzed by comparing the first (2001-2003) with the last time period (2015-2017). In general, men and women were equally likely to use psychotropic drugs. Monotherapy was more common in men. Women were more likely to utilize $\geq 4$ psychotropic drugs. Antidepressant drugs (ADDs) were the most prescribed drug class. Men had a higher utilization of noradrenergic and specific serotonergic antidepressants (RR 1.15; 95\% CI 1.12-1.19), especially mirtazapine (RR 1.16; 95\% CI 1.12-1.19), but also of other ADDs such as bupropion (RR 1.50; 95\% CI 1.35-1.68). Males had a slightly higher utilization of secondgeneration antipsychotic drugs (RR 1.06; 95\% CI 1.03-1.09) and were less often treated with low-potency first-generation antipsychotic drugs (RR 0.86; 95\% CI 0.83-0.90). Tranquilizing (e.g., benzodiazepines; RR 0.89; 95\% CI 0.86-0.92) and hypnotic drugs (e.g., Z-drugs; RR 0.85; 95\% CI 0.81-0.89) were less utilized in the treatment of male patients. Not all sex differences were stable over time. More sex differences were detectable in 2015-2017 than in 2001-2003. Findings suggest that certain psychotropic drugs are preferred in the treatment of men vs. women, however, sex differences found in this study are not as large as in ambulatory settings. To make evidence-based sex-specific recommendations in the treatment of MDD, differences in drug response and tolerability need to be further researched.
\end{abstract}

Keywords Antidepressant drugs $\cdot$ Antipsychotic drugs $\cdot$ Drug safety $\cdot$ Psychopharmacotherapy $\cdot$ Gender $\cdot$ Depression

Johanna Seifert and Fabienne Führmann are contributed equally as first authors, Marcel Sieberer and Waldemar Greil are contributed equally as senior authors.

Johanna Seifert

seifert.johanna@mh-hannover.de

1 Department of Psychiatry, Social Psychiatry, and Psychotherapy, Hannover Medical School, Carl-Neuberg-Straße 1, 30625 Hannover, Germany

2 Department of Psychiatry and Psychotherapy, KRH Psychiatrie GmbH, Wunstorf, Germany

3 Department of Psychiatry and Psychotherapy, LMU University Hospital Munich, Munich, Germany

4 Department of Forensic Psychiatry, Bezirksklinikum Ansbach, Ansbach, Germany
5 Department of Psychiatry, Psychotherapy, and Psychosomatics, St. Marien-Hospital Hamm gGmbH, Hamm, Germany

6 Prosomno, Clinic for Sleep Medicine, Munich, Germany

7 Department of Psychiatry and Psychotherapy, University Witten/Herdecke, Witten, Germany

8 Psychiatric Private Hospital, Sanatorium Kilchberg, Kilchberg, Switzerland 


\section{Introduction}

Incidence of depression worldwide has increased by nearly $50 \%$ within the past three decades (Liu et al. 2019) with women remaining twice as likely to suffer from depression (Herzog et al. 2019). Women suffering from major depressive disorder (MDD) are more likely to have an earlier age of onset, develop subsequent depressive episodes, and present a chronic course of illness (Frackiewicz et al. 2000). Studies consistently report a higher utilization of antidepressant drugs (ADDs) among women within the ambulatory setting (Boyd et al. 2015; Sundell et al. 2011; Zhong et al. 2014; Serna et al. 2010; Van der Heyden et al. 2009; Estancial Fernandes et al. 2018). However, this pattern may not necessarily represent the psychiatric inpatient setting.

Biologically determined sex differences in brain and physiology play a relevant role in the development of MDD (Rubinow and Schmidt 2019), expression of clinical symptoms (Altemus et al. 2014) as well as in efficacy and tolerability of drugs used to treat MDD (Franconi and Campesi 2014; LeGates et al. 2019). The latter are currently not fully understood, in part due to a low rate of women's participation in clinical trials in the past (Liu and Mager 2016). In the past, sex differences were largely ignored-the exclusion of women justified due to the complexity of the female hormonal cycle which would complicate investigations (Rubinow and Schmidt 2019). First implications of sex differences in the treatment of MDD arose in the late 1960s when $\mathrm{T}_{3}$ (L-triiodthyronine) was observed to be more effective in the augmentation of antidepressant treatment in women than men-a finding which encouraged further research (Khan et al. 2005). In the past decades, sex has been finding more extensive consideration in the treatment of MDD (Khan et al. 2005).

As of now, standardized guidelines on the sex-specific treatment of MDD are virtually unavailable. A recent review about sex differences in antidepressant response by LeGates et al. summarized that there is not a definite consensus on whether sex differences in antidepressant efficacy actually exist (LeGates et al. 2019). International guidelines on the treatment of MDD, such as the recommendations by the British Association for Psychopharmacology or the German S3 guideline by the German Association for Psychiatry, Psychotherapy, and Psychosomatics, merely suggest that women may benefit more from treatment with a selective serotonin reuptake inhibitor (SSRI) (DGPPN et al. 2017), while men may respond better to treatment with tricyclic antidepressants (TCAs) (Cleare et al. 2015; DGPPN et al. 2017). The National Institute for Health and Care Excellence (NICE) explicitly states, that little evidence supports prescribing patterns in relation to sex ((NICE) 2009).
The aim of this study was to assess the use of psychotropic drugs used in the treatment of patients suffering from MDD according to sex in a real-life clinical inpatient setting from 2001 to 2017. Because treatment of MDD is not limited to the use of ADDs, utilization of other psychotropic drug classes (i.e., antipsychotic drugs (APDs), antiepileptic drugs (AEDs), lithium (LI), tranquilizing drugs (TRDs), and hypnotic drugs (HYPDs)) and combination of drug classes will also find consideration. Furthermore, time trends in the sex-specific treatment of MDD are analyzed by comparing the first (2001-2003) with the last (2015-2017) time period to provide information on more recent utilization patterns and determine the temporal stability of sex differences.

\section{Methods}

\section{Data source}

This study used data pertaining to the utilization of psychotropic drugs by patients with MDD collected by the European program "Drug Safety in Psychiatry" (German: "Arzneimittelsicherheit in der Psychiatrie", AMSP). Founded in 1993, AMSP has since gathered data on psychotropic drug use and severe adverse drug reactions (ADRs) from psychiatric hospitals within a real-life setting. The number of participating hospitals has increased from nine in 1994 to 52 psychiatric institutions in Germany, Austria, and Switzerland in 2017.

Drug use data are gathered on two reference days per year on which all participating hospitals document all drugs prescribed on these days including dosages along with further information on age, sex, as well as psychiatric and somatic illnesses of patients. Due to the inpatient setting, AMSP is able to assess actual utilization rates of psychotropic drugs versus merely prescription rates. A more detailed description of AMSP's methods can be found elsewhere (Grohmann et al. 2004, 2014; Engel et al. 2004). Data evaluation and analysis of the AMSP database have been approved by both the Ethics Committee of the University of Munich and the Ethics Committee of the Hannover Medical School (Nr. 8100_BO_S_2018).

\section{Study population and design}

All patients treated between 2001 and 2017 aged 18-100 years with a primary psychiatric diagnosis of MDD were included in the study. Other psychiatric comorbidities, such as anxiety disorders, PTSD, or substance abuse disorders, were not considered during analysis of data. Drug utilization data by sex on the reference days were included in further analyses. MDD was identified using the International Classification of Disease in its 10th Version (ICD-10) (WHO 
$1992)$ and categorized as mild (F32.00, F32.01, F33.0, F33.00, F33.01), moderate (F32.1, F32.10, F32.11, F33.1, F33.10, F33.11), and severe depression without (F32.2, F33.2) or with psychotic symptoms (F32.3, F33.3). By including all degrees of severity, this study aims to provide a comprehensive insight in the psychopharmacological treatment of all inpatients with MDD. Prior to 2018 - and therefore including the entire data collection period-patients suffering from non-severe depression could receive inpatient care. This later changed after implementation of a new remuneration system which led to a tightening of admission criteria.

\section{Classification of psychotropic drugs}

ADDs were categorized as follows:

- SSRIs: escitalopram, citalopram, sertraline*

- Selective serotonin-norepinephrine reuptake inhibitors (SSNRIs): venlafaxine, duloxetine*

- TCAs: trimipramine, amitriptyline, doxepin*

- Noradrenergic and specific serotonergic antidepressants (NaSSAs): mirtazapine*

- Monoamine oxidase inhibitors (MAOIs): tranylcypromine, moclobemide**

- Other ADDs: trazodone, bupropion, agomelatine*

APDs were classified as "first-generation antipsychotics" (FGAs) or "second-generation antipsychotics" (SGAs). FGAs were sub-classified as "low potency" (lp) or "high potency" (hp).

- lp FGAs: pipamperone, promethazine, prothipendyl, melperone*

- hp FGAs: haloperidol, perazine, flupentixol**

- SGAs: quetiapine, olanzapine, risperidone, aripiprazole*

HYPDs primarily included the Z-drugs (zopiclone, zolpidem*). The group of TRDs mainly consisted of benzodiazepines (especially lorazepam, diazepam, oxazepam*). Finally, relevant AEDs included valproic acid, lamotrigine, and pregabalin*.

$*$ Only drugs used in the treatment of $\geq 2.5 \%$ patients are listed.

**Because of very low overall utilization in these drug groups, drugs used in the treatment of $\geq 0.5 \%$ patients are listed.

\section{Statistical analysis}

Sex-specific drug utilization was analyzed by calculating relative utilization rates between sexes as relative risks (RR) together with their 95\% confidence intervals $(95 \%$
CI). Changes in the sex-specific use of drugs over time were analyzed for two observation periods, namely 2001-2003 and 2015-2017. In addition to time-specific RRs, relative risk ratios (RRR) were calculated with their $95 \%$ confidence intervals to quantify the interaction term sex by time. RRs and RRRs were considered statistically significant when the null value (i.e., 1.0) was not included in the $95 \%$ CI.

\section{Results}

\section{Sociodemographic and illness-related data according to sex}

44,418 patients with a primary diagnosis of MDD were treated in the participating hospitals from 2001 to 2017. $62.7 \%$ of patients were female. Table 1 shows the distribution of characteristics (i.e., age groups, severity of MDD, number of psychotropic drugs) between sexes. Women were older than men ( $q: 51.53$ vs. $\delta^{\lambda}: 48.81$ years). Severity of MDD showed only slight differences between sexes. Most common diagnosis among both men and women was severe depression without psychotic symptoms ( $q: 58.6 \%$;

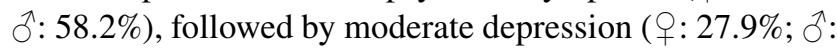
$27.5 \%$ ), and severe depression with psychotic symptoms ( $($ : $12.4 \%$; $12.9 \%)$. The diagnosis of mild depression was rare in this sample of inpatients. Most patients ( $(9: 96.2 \%$; $\overbrace{}^{\top}$ : 95.3\%) were treated with psychotropic drugs. Men were 23\% more likely not to receive any psychotropic medication and 9\% less likely to be treated with four or more psychotropic drugs than women (Table 1).

\section{Prescription trends of psychotropic drugs according to sex}

\section{Antidepressant drugs}

ADDs were the most used psychotropic drug class; however, they were used less often in the treatment of both men and women in 2015-2017 ( $\delta: 84.1 \%$; $9: 86.4 \%$ ) than in 2001-2003 (: 89.1\%; 0 : 90.0\%). NaSSAs-mainly consisting of mirtazapine-were the ADD-subgroup with the greatest sex difference in utilization rates. NaSSAs were used more frequently in the treatment of men. In 2001-2003, use of mirtazapine was equal among both sexes, whereas utilization rates of mirtazapine were relevantly higher among male patients in 2015-2017 (Table 2).

Overall utilization of SSRIs was minimally higher among females, in particular in 2015-2017. This trend was especially noticeable for escitalopram, which was used more often in the treatment of MDD in 2015-2017 than 2001-2003 in general, and especially among women. Use of SSNRIs as a group did not show relevant sex differences at 
Table 1 Characteristics of the study population according to sex

\begin{tabular}{|c|c|c|c|c|c|}
\hline & \multicolumn{2}{|l|}{ M } & \multicolumn{2}{|l|}{$\mathrm{F}$} & \multirow[t]{2}{*}{ RR $(95 \%$ CI $)$} \\
\hline & $N$ & $(\%)$ & $N$ & $(\%)$ & \\
\hline Total & 16,547 & 37.3 & 27,871 & 62.7 & \\
\hline Age in years (mean) & 48.81 & & 51.53 & & \\
\hline \multicolumn{6}{|l|}{ Age groups } \\
\hline$<31$ & 2466 & 14.9 & 3585 & 12.9 & $1.15(1.10-1.22)$ \\
\hline $31-60$ & 10,396 & 62.8 & 16,019 & 57.5 & $1.09(1.08-1.11)$ \\
\hline $61-90$ & 3661 & 22.1 & 8197 & 29.4 & $0.75(0.73-0.78)$ \\
\hline$>91$ & 24 & 0.1 & 70 & 0.3 & $0.58(0.36-0.92)$ \\
\hline \multicolumn{6}{|l|}{ Diagnosis } \\
\hline Mild depression & 236 & 1.4 & 314 & 1.1 & $1.27(1.07-1.50)$ \\
\hline Moderate depression & 4543 & 27.5 & 7773 & 27.9 & $0.98(0.95-1.02)$ \\
\hline Severe depression & 9636 & 58.2 & 16,326 & 58.6 & $0.99(0.98-1.01)$ \\
\hline Severe depression with psychosis & 2132 & 12.9 & 3458 & 12.4 & $1.04(0.99-1.09)$ \\
\hline Pat. receiving any psychotropic drugs & 15,764 & 95.3 & 26,799 & 96.2 & $0.99(0.99-0.99)$ \\
\hline N psychotropic drugs (mean) & 2.60 & & 2.68 & & \\
\hline \multicolumn{6}{|l|}{ N psychotropic drugs } \\
\hline 0 & 783 & 4.7 & 1072 & 3.8 & $1.23(1.12-1.35)$ \\
\hline 1 & 3553 & 21.5 & 5527 & 19.8 & $1.08(1.04-1.12)$ \\
\hline 2 & 4923 & 29.8 & 8246 & 29.6 & $1.00(0.98-1.03)$ \\
\hline 3 & 3847 & 23.2 & 6690 & 24.0 & $0.97(0.94-1.00)$ \\
\hline $4+$ & 3441 & 20.8 & 6336 & 22.7 & $0.91(0.88-0.95)$ \\
\hline
\end{tabular}

$N$ number, $M$ males, $F$ females, $d f$ degrees of freedom, pat. patients, $R R$ relative risk, $95 \% C I 95 \%$ confidence interval any time point. Duloxetine was used $13 \%$ less frequently in the treatment of men than women in 2001-2017 (Table 2).

Use of "other ADDs" greatly increased from 2001-2003 to 2015-2017. Within this heterogeneous group, trazodone was the most used drug. Rarely used in 2001-2003, trazodone was more commonly utilized in 2015-2017, especially among women. Data for bupropion and agomelatine were only available for the later timeframe. From 2015 to 2017, utilization of bupropion was almost 1.5 times higher among men, while men were less likely to be treated with agomelatine (Table 2).

TCAs were used slightly more often in the treatment of women from 2001 to 2017. Sex-specific utilization and time trends for MAOIs as well as for other individual ADDs can be found in Table 2 .

\section{Antipsychotic drugs}

Almost half of men and women were treated with APDs from 2001 to 2017 ( $\lesssim: 49.3 \%$; : $49.4 \%$ ). APD-use was slightly higher among men in 2015-2017. Men were more likely to be treated with SGAs - this trend was especially apparent in 2001-2003 at which time SGAs were used in the treatment of $30.1 \%$ of men and $24.7 \%$ of women. In 2015-2017, SGA utilization rates were higher among both sexes (ठ: 41.1\%; 吊: 37.4\%) than in 2001-2003 but did not differ as much between sexes. Similar patterns were found for the use of olanzapine. Utilization of quetiapine was low in 2001-2003, whereas its utilization was 6.5 times higher among women and eightfold higher among men in 2015-2017. At this time, men were more likely to be treated with quetiapine (Table 3).

Utilization of lp FGAs in general as well as of the four most commonly used individual substances (i.e., pipamperone, promethazine, prothipendyl, melperone) was lower in men overall as well as in both time periods. Use of hp FGAs did not show any relevant sex differences (Table 3 ).

\section{Tranquilizing and hypnotic drugs}

TRDs were the third-most utilized drug class. Men were $11 \%$ less likely to be treated with TRDs ( $ત: 27.2 \%$; 9 : $30.5 \%$ ). TRD use decreased from 2001-2003 to 2015-2017. While in 2001-2003, utilization of TRD did not show a sex difference, women had slightly higher utilization rates in 2015-2017. Lorazepam was the most prescribed TRD, also with lower use among male patients. Use of diazepam, much less used than lorazepam overall, further decreased in 2015-2017 and was used more by men (Table 4).

HYPDs were the fourth-most used drug class from 2001 to 2017 and were used less frequently in the treatment of 


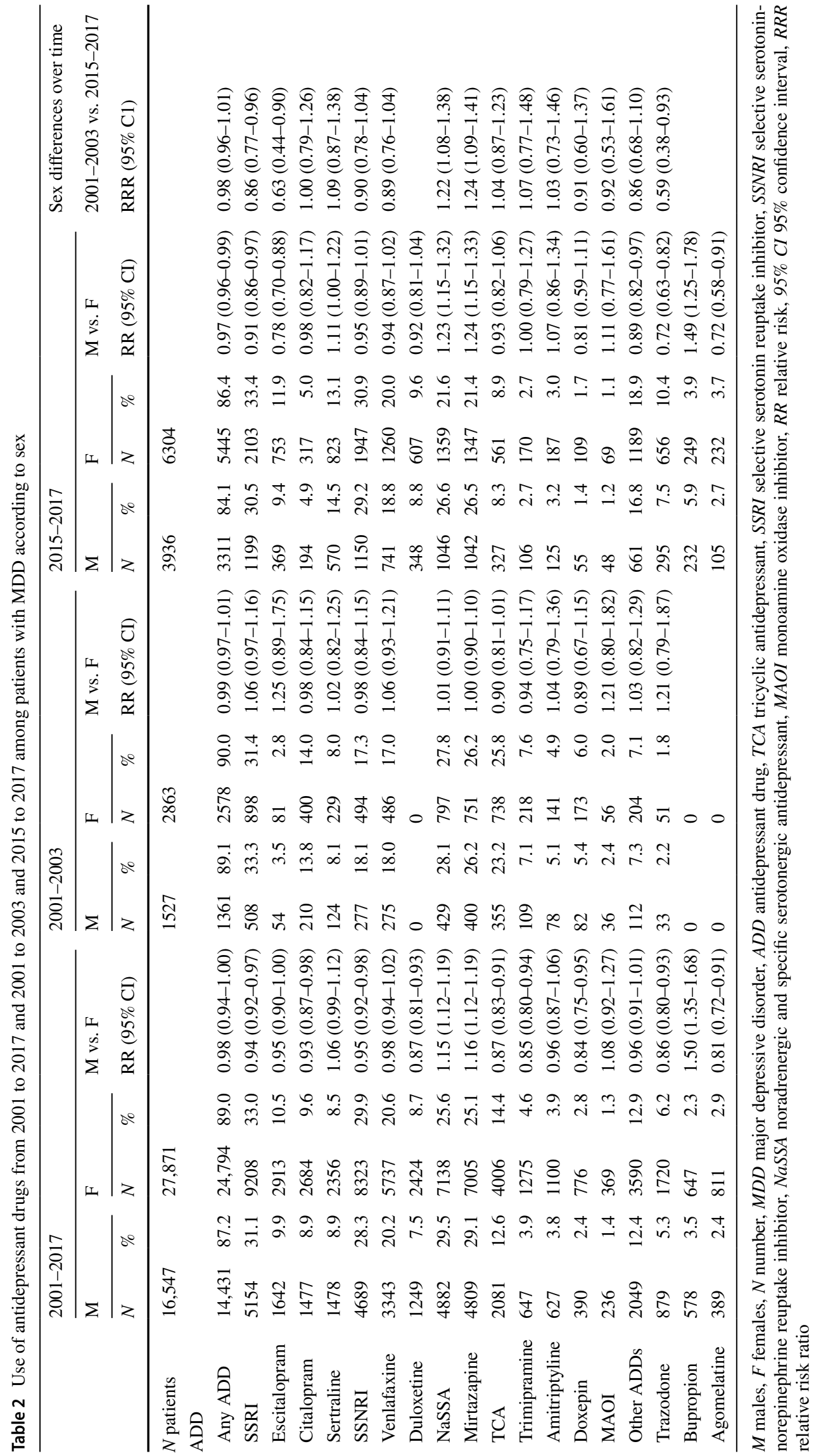




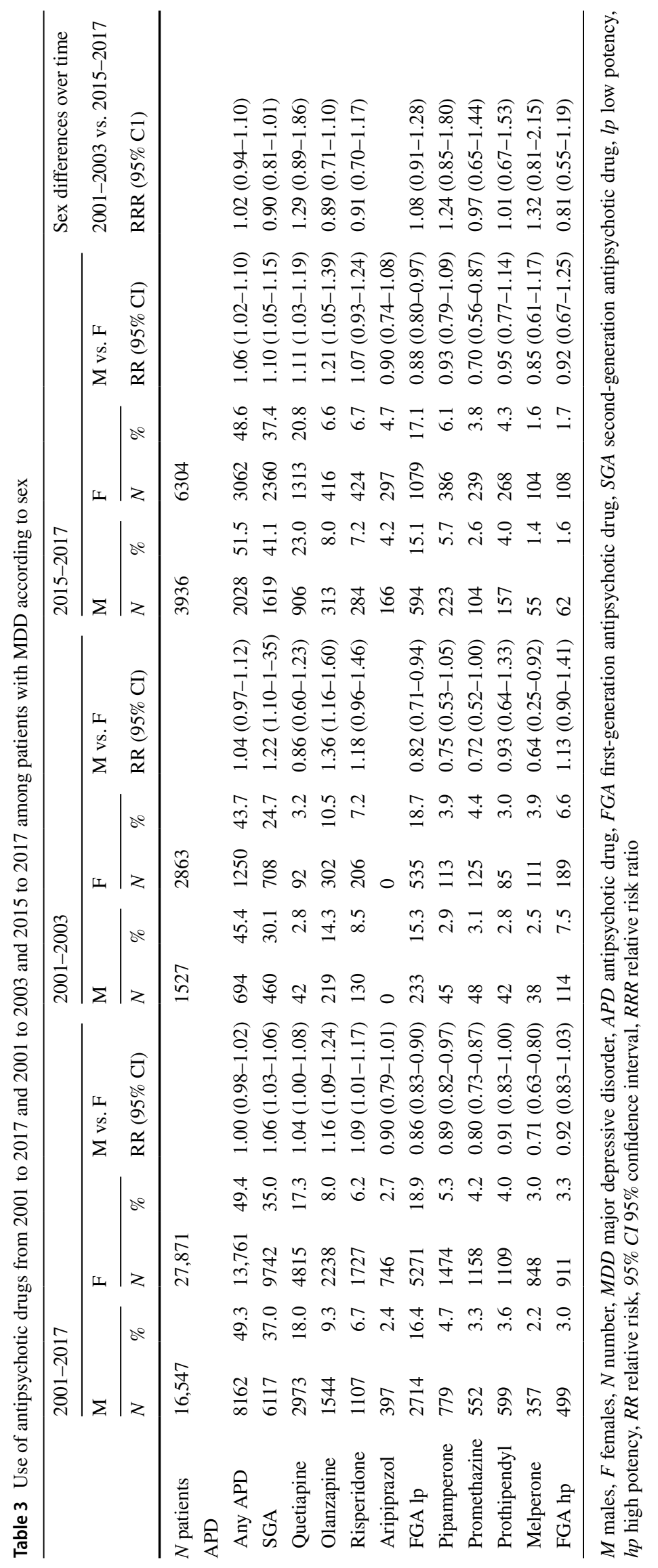




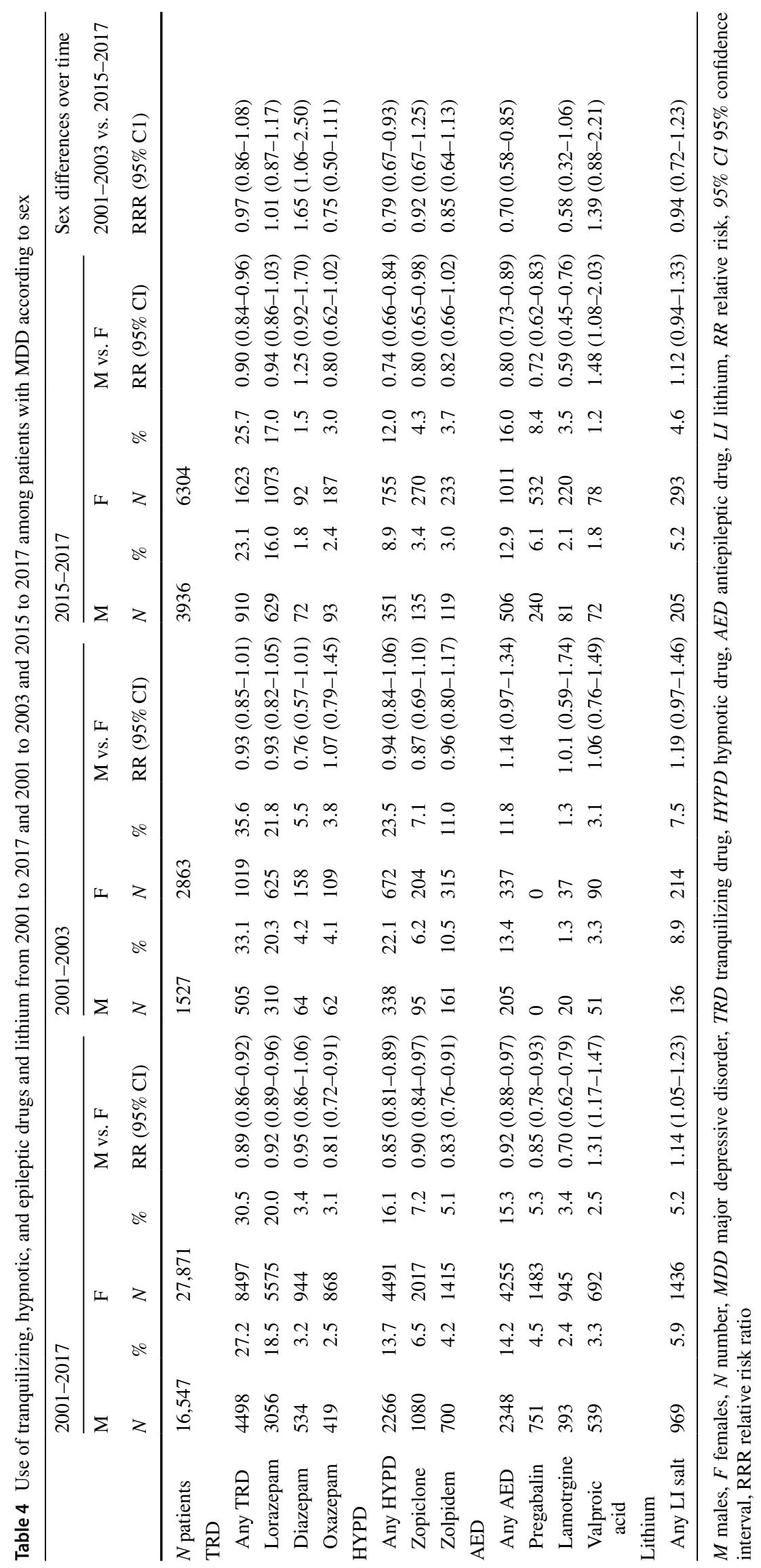


men. Sex-related use of HYPDs did not differ in 2001-2003. In 2015-2017, men had a 26\% lower HYPD utilization than women and therefore comprised the drug class with the greatest sex difference in 2015-2017 (Table 4).

\section{Antiepileptic drugs and lithium}

Overall AED utilization was slightly higher among females, especially in the later time period. In 2015-2017, men were less likely to be treated with lamotrigine and pregabalin. The sex ratio was reversed for the use of lithium. Lithium salts were the least utilized psychotropic drug class. Men had $14 \%$ higher utilization rates of lithium from 2001 to 2017 (Table 4).

\section{Trends in polypsychopharmacy}

Concurrent utilization of ADD + APD was the most common drug combination among men and women and used by half of all male and female ADD users ( $\delta$ : $50.3 \%$; $50.1 \%)$. ADD + APD was slightly more often used in treatment of men in 2015-2017. Second-most common combination was the concomitant utilization of two ADDs ( $\delta$ : $32.0 \%$; + : $31.1 \%)$ which did not show any relevant sexspecific trends. Use of ADD + TRD ( ${ }^{\lambda}: 28.0 \%$; 우: $31.0 \%$ ) was the third-most common drug combination. Utilization of ADD + TRD was higher among females from 2001 to 2017 and 2015 to 2017. The combination of HYPDs and AEDs with ADDs both showed a higher utilization among women in 2015-2017 without any clear sex differences in 2001-2003 (Table 5).

\section{ADD and concomitant utilization of Ip FGAs}

Concomitant use of lp FGAs with different ADD-subgroups was lower among men during the overall observation period, especially for lp FGA + SSRI and lp FGA + NaSSA. However, sex differences for $1 p$ FGA + SSRI and $1 p$ FGA + NaSSA were no longer detectable in 2015-2017; thus, this drug combination had the most significant change in sex differences from 2001-2003 to 2015-2017. In 2015-2017, use of lp FGA + SSNRI was the only combination of ADD-subgroup with lp FGAs with a sex difference showing higher utilization among women (Table 6).

\section{ADD and concomitant utilization of SGAs}

Utilization of SGA + ADD increased for all ADD subgroups (i.e., SSRIs, SSNRIs, NaSSAs) from 2001-2003 to 2015-2017. Concomitant treatment with an SGA was highest among SSNRI users during both the entire observation period, 2001-2003, and 2015-2017. This combination was used more in male patients overall and in 2001-2003 but no longer in 2015-2017. Use of SSRI + SGA was used more in the treatment of men in 2001-2003 and 2015-2017 whereas no sex differences were observed for NaSSA + SGA (Table 6).

\section{Concomitant utilization of two antidepressant drugs}

Male SSNRI users were 36\% more likely to also be treated with NaSSAs than females from 2001 to 2017. This was also the case in 2015-2017. The combined use of SSRI+ NaSSA did not show a sex difference from 2001 to 2017. However, this combination of ADDs was used more in the treatment of male SSRI users than females in 2015-2017 (Table 6).

\section{ADD and concomitant utilization of TRDs and HYPDs}

When examining the overall time period, concomitant use of TRDs was $9 \%$ lower among male NaSSA users and $18 \%$ lower among male SSRI users. Sex differences were not detectable in 2001-2003 or 2015-2017. Utilization of SSNRI + TRD did not show sex differences overall or at any time point (Table 6).

From 2001 to 2017, HYPD use was 29\% lower among lower among male NaSSA users and 22\% lower among male SSRI users. Concomitant use of HYPDs with SSRIs, SSNRIs, and NaSSAs was higher among female patients in 2015-2017. In 2001-2003, use of these drug combinations did not differ between sexes. SSRI + HYPD was the combination with the most prominent sex difference (Table 6).

\section{Most used combinations of two psychotropic drugs}

The most frequently used combination and with a 7\% higher utilization among men was mirtazapine + lorazepam (2802 patients from 2001 to 2017 , i.e., $22.7 \%$ of male mirtazapine users vs. $24.4 \%$ of female mirtazapine users; RR 0.93; $95 \%$ CI 0.87-0.99). Second-most common was the concomitant use of venlafaxine + mirtazapine (2270 patients; i.e., $28.1 \%$ of male venlafaxine users vs. $23.2 \%$ of female venlafaxine users). Male venlafaxine users were $21 \%$ more likely to receive this drug combination (95\% CI 1.13-1.30). A sex difference was not detectable when considering this combination from the perspective of mirtazapine users-venlafaxine was concomitantly used by $19.5 \%$ of male and $19.0 \%$ of female mirtazapine users. Third-most common drug combination was venlafaxine + quetiapine (2002 patients) used by $22.0 \%$ and $22.1 \%$ of male and female venlafaxine users, respectively.

\section{Most used combination of three psychotropic drugs}

Nearly one-fourth of women and men were treated with three psychotropic drugs (Table 1). The most common triple 


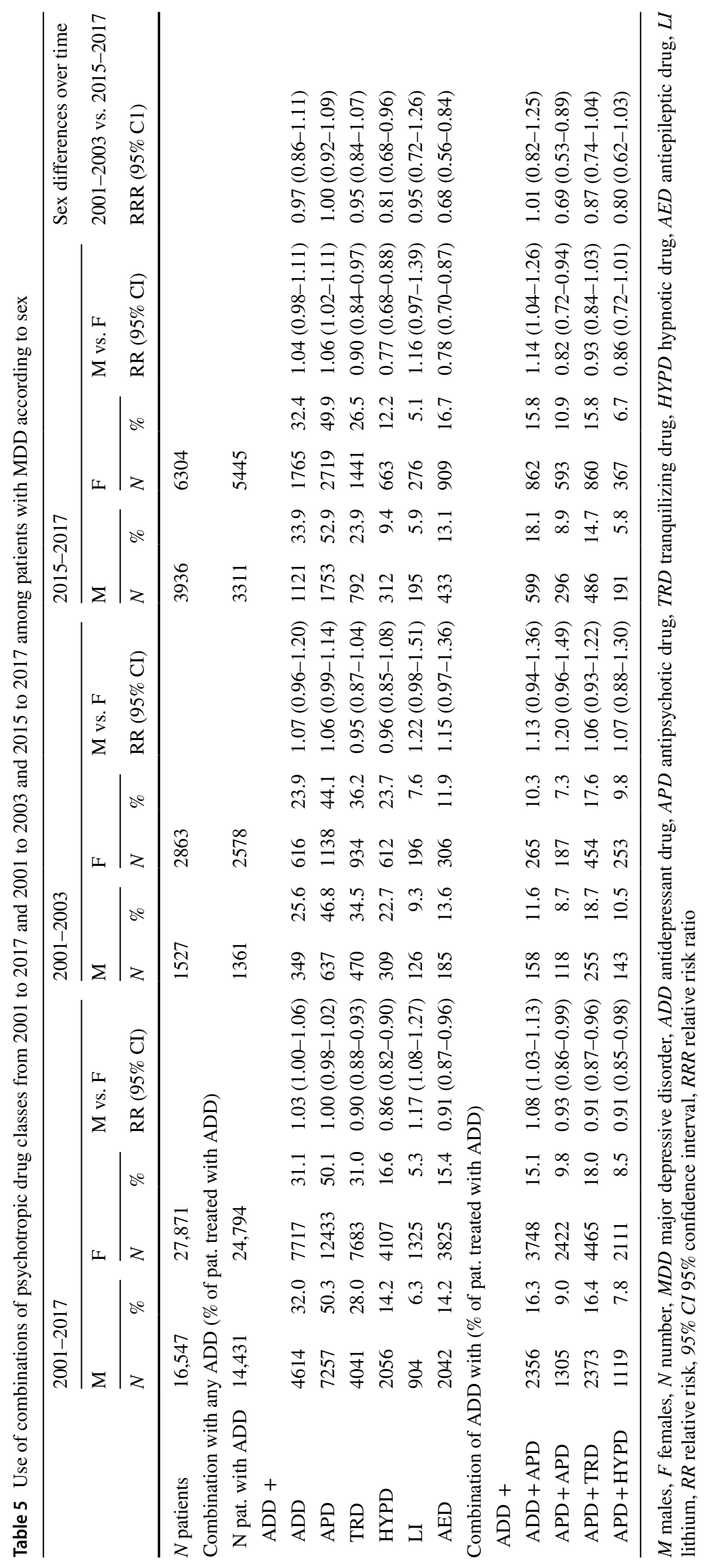




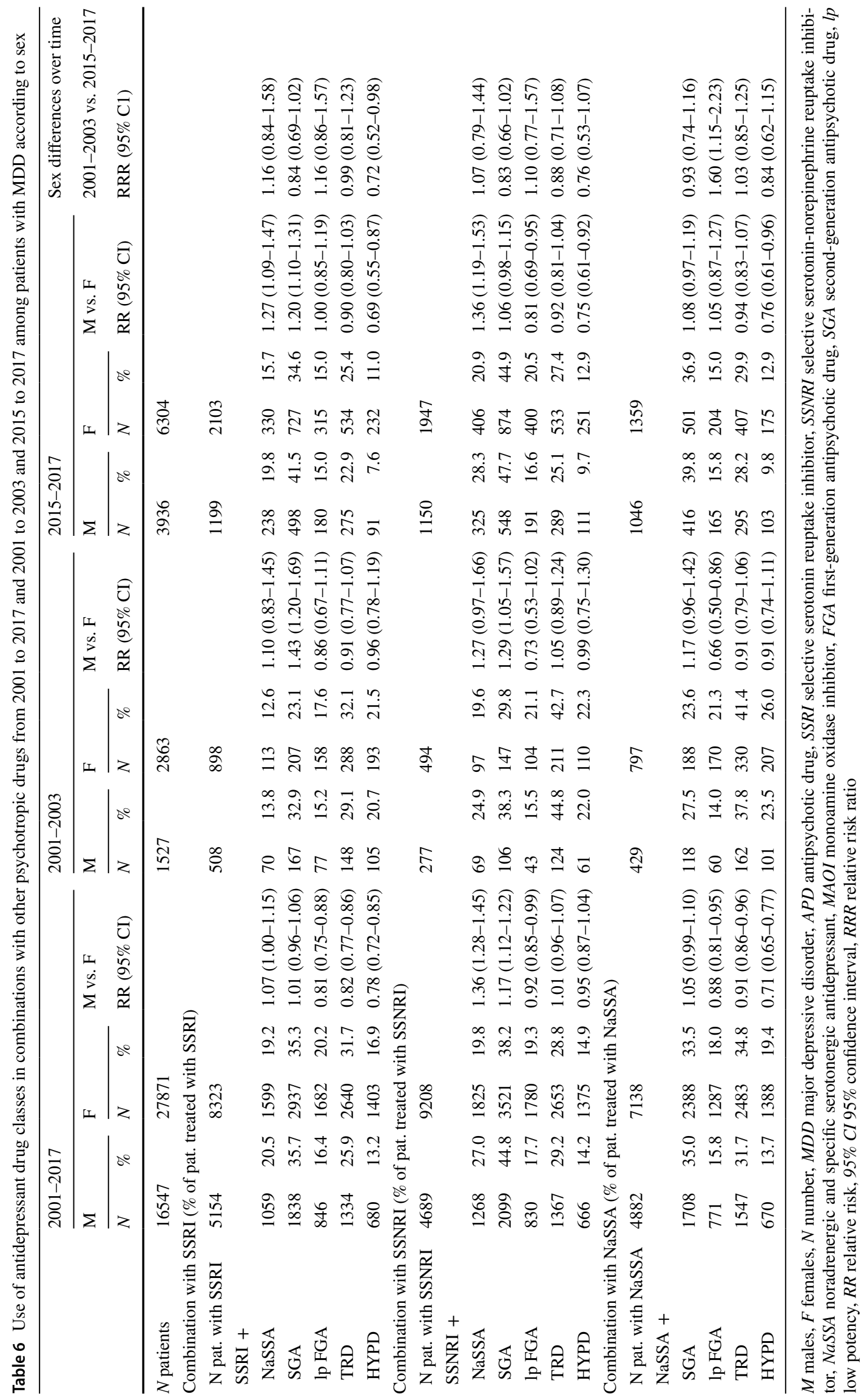


psychotropic drug class combination among both men and women was ADD + APD + TRD. Men had a lower risk of being treated with this combination in 2001-2017, however, utilization of this drug group combination was equal among men and women in 2015-2017. The second-most common triple combination among both sexes was the use of two ADDs and an APD—a drug combination more frequently used in the treatment of male ADD users than females. This combination was more utilized in 2015-2017, at which time utilization was also higher among men (Table 5).

Venlafaxine, mirtazapine, and lorazepam were the most used combination of individual drugs ( 605 patients; i.e., $7.3 \%$ of male venlafaxine users vs. $6.3 \%$ of female users or $5.1 \%$ of male mirtazapine users vs. $5.2 \%$ of female users). Mirtazapine, quetiapine, and lorazepam were used by 570 patients (4.9\% of male mirtazapine users vs. $4.8 \%$ of female users), followed by venlafaxine, mirtazapine, and quetiapine (500 patients; i.e., $4.6 \%$ of male mirtazapine users vs. $4.0 \%$ of female users or $6.6 \%$ of male venlafaxine users vs. $4.9 \%$ of female users). None of the most common triple combinations showed relevant sex differences.

\section{Discussion}

The present study focuses on sex differences of psychotropic drug utilization in the treatment of psychiatric inpatients with MDD over a 17-year period. In order to detect time trends in sex-specific drug utilization, the timeframes 2001-2003 and 2015-2017 were also analyzed. To the best of our knowledge, this is the first study investigating drug utilization trends of patients with MDD with special attention to sex differences over time within the inpatient psychiatric setting. A more detailed analysis of time trends in the utilization of psychotropic drugs during this time period can be found elsewhere (Seifert et al. 2021). In brief, overall utilization of ADDs decreased slightly during this timeframe. TCAs were used less frequently in 2015-2017, whereas utilization of SSRIs and "other ADDs" increased. More patients were treated with a combination of two ADDs in 2015-2017 than 2001-2003. Further, APDs, especially SGAs, appeared to "replace" the use of TRDs and HYPDs (Seifert et al. 2021).

\section{Sex differences in psychotropic drug utilization}

Utilization of psychotropic drugs is associated with a patient's likeliness to seek medical care (Subramaniam et al. 2013), a factor that has been eliminated in inpatient setting in which treatment has already been sought. The present study was able to confirm some of the observations other researchers have made, however, relative risks for the use of psychotropic drug groups were usually not as discrepant among this collective of inpatients as among the outpatient collectives analyzed by other authors. For example, while Serna et al. found that Spanish men were about $60 \%$ less likely to use any psychotropic drug than women (Serna et al. 2010), over 95\% of both male and female inpatients examined in this study were treated with at least one psychotropic drug. A majority of currently available studies have found higher psychotropic drug utilization among women within the ambulatory setting (Sundell et al. 2011; Luo et al. 2020; Zhong et al. 2014; Estancial Fernandes et al. 2018; Serna et al. 2010; Boyd et al. 2015) - a plausible finding when considering that common mental disorders such as MDD are more prevalent in women (Herzog et al. 2019). The high utilization of psychotropic drugs among inpatients allows a more differentiated look at sex-related utilization trends of psychotropic drug classes and individual drugs, which previous studies have rarely commented on.

Population-based RRs for the utilization of ADDs (Boyd et al. 2015; Van der Heyden et al. 2009; Estancial Fernandes et al. 2018; Sundell et al. 2011; Zhong et al. 2014; Serna et al. 2010; Luo et al. 2020; Yu et al. 2020) by women has been noted to be up to 2.42 (Serna et al. 2010) times higher than by men. Studies considering subclasses of ADDs found that women were more likely to be treated with SSRIs (Sundell et al. 2011; González-López et al. 2015) and TCAs (Sundell et al. 2011). The study which presents the most detailed description of sex-specific drug utilization patterns among outpatients with MDD was performed by Sundell et al. They found that citalopram and sertraline followed by venlafaxine and mirtazapine were the most frequently prescribed ADDs among a Swedish outpatient population of young adults aged 20-34 years. Men were more than twice as likely to be prescribed mirtazapine (RR 2.22), while women had a $10 \%$ higher chance of being prescribed SSRIs, especially fluoxetine (RR 1.70), and were $14 \%$ more likely to use TCAs. Among SSNRIs, use of duloxetine was 13\% lower among men, whereas men had $21 \%$ higher odds of being prescribed venlafaxine (Sundell et al. 2011).

In the present study, the greatest sex difference in ADDutilization was found for NaSSAs. NaSSAs were also the only ADD subgroup which were used more often in the treatment of men than women (RR 1.16 from 2001 to 2017). Female patients were treated with TCAs more often during the overall observation period, however, this trend was not detectable at either time point. Utilization of SSRIs was higher among women during the later time period, whereas a sex difference was not detectable in 2001-2003. The shift to higher utilization of SSRIs in the treatment of women is potentially due to the application of the German S3 guidelines in which the higher efficacy of SSRIs in the treatment of women is mentioned without being definitively recommended (DGPPN et al. 2017). 
Sex differences in the use of anxiolytic drugs are reported inconsistently. Several authors were unable to detect significant sex differences in the dispensation of benzodiazepines (Van der Heyden et al. 2009; Estancial Fernandes et al. 2018; Subramaniam et al. 2013). Among the general population, Boyd et al. found German women were more likely to be treated with benzodiazepines. When adjusted for prevalence of anxiety disorders and MDD, utilization of benzodiazepines did not show any sex differences in the German ambulatory setting (Boyd et al. 2015). Higher benzodiazepine use was found among Portuguese, Bulgarian, French, and Spanish women with MDD (Boyd et al. 2015). The results presented here show that utilization of HYPDs and TRDs has shown significant changes during this study's 17 -year observation period. Use of both drug groups has decreased from 2001-2003 to 2015-2017. Synchronously, sex differences have appeared. In more recent years, women have been more likely to be treated with these drug groups, especially HYPDs.

Sex differences for the prescription of APDs and moodstabilizing drugs, such as AEDs and lithium, among patients with MDD are largely unavailable. A study examining the population-based prevalence of psychotropic drug use over a 12-month period in ten European countries found that the use of APDs and mood-stabilizing drugs did not show any significant sex differences among patients with mood disorders in any country (Boyd et al. 2015). Results presented in this study show that alongside a decline in TRD and HYPD utilization from 2001-2003 to 2015-2017, overall use of APDs increased. This may signify that TRDs and HYPDs are being replaced by other psychotropic drug classes that do not carry a risk for dependency. This seems to be the case especially among male patients. While TRD and HYPD use was not sex-dependent in 2001-2003, this study revealed in particular a relevantly higher (i.e., 25\%) HYPD utilization among women in 2015-2017. Compared to this, APD utilization among men only showed a disproportionately small increase (i.e., 6\%) indicating that there may also be further underlying considerations, such as ADRs, that decidedly affect selection of drug prescriptions.

Sundell et al. found that men were significantly more likely to be treated with AEDs, such as carbamazepine, lamotrigine, and valproic acid, whereas the use of lithium showed no sex-related trends (Sundell et al. 2011). Higher use of lithium has been described among male patients with bipolar disorder, while women were more often treated with lamotrigine (Karanti et al. 2015). The same study found similar prescription rates of APDs and other mood-stabilizing agents including valproic acid among sexes (Karanti et al. 2015). In this study, valproic acid showed a very prominent utilization trend of preferred use among male patientsa finding that is not surprising under consideration of the drug's teratogenic properties as well as the risk for hormonal abnormalities (Gotlib et al. 2017). This sex-specific recommendation found more consideration in 2015-2017 at which point utilization of valproic acid was lower and utilization of lamotrigine was higher in the treatment of female patients.

\section{Sex differences in response to psychotropic drugs}

Undeterred by years of research, recommendations for sexspecific treatment of MDD are largely speculative. While many authors are unable to detect any sex-based differences of the efficacy of ADDs, others state superiority when used in either males or females. However, findings are inconsistent (Sramek et al. 2016). It appears that SSRIs and to a lesser extent SSNRIs may be superior in the treatment of women. Men, on the other hand, may respond better to TCAs (Sramek et al. 2016). In a meta-analysis including 15 studies, Khan et al. examined sex-specific treatment response. While both sexes showed significant improvement, women improved to a greater extent than men when treated with an SSRI, while treatment with an SSNRI showed similar responses among sexes (Khan et al. 2005). Unfortunately, most of these studies do not take the occurrence of ADRs into account (Sramek et al. 2016) which are often pivotal in the acceptance of any medication (Sansone and Sansone 2012).

Serotonergic ADRs often caused by SSRIs and SSNRIs may induce or worsen pre-existing sexual dysfunction (Montejo et al. 2019b). While sexual dysfunction as an ADR affects men and women equally, it may be more poorly tolerated by men (Montejo et al. 2019a) resulting in cessation of the drug or decreased willingness to start treatment in the first place. Bupropion and mirtazapine are associated with a lower incidence of sexual dysfunction (Montejo et al. 2019b) and may therefore be preferred by men. This may in part explain the higher utilization of mirtazapine, and even more significantly bupropion, as found in this study.

Higher utilization of lp FGAs by women and SGAs by men was consistent both in 2001-2017 and 2015-2017. A reason for this may also be the underlying clinical considerations regarding potential ADRs. Weight gain is frequently associated with certain psychotropic drugs, especially SGAs, such as olanzapine and quetiapine, but also ADDs, such as mirtazapine (Schneider et al. 2020), which each showed higher utilization among men at some point during this study's observation period. Mirtazapine and olanzapine showed sex differences during the overall observation period. Initially in 2001-2003, mirtazapine had similar utilization rates among sexes with significant differences in utilization in 2015-2017. At this time, quetiapine also showed higher use among men, which was not the case in the overall observation period or in 2001-2003. Among drugs with the 
greatest risk for weight gain, quetiapine showed the smallest sex difference overall and also in 2015-2017, perhaps because quetiapine (extended release) is the only APD that is in-label for the treatment of MDD according to German guidelines (DGPPN et al., 2017).

Parallel to the increased use of weight gain-inducing psychotropic drugs by men, utilization of HYPDs and TRDs showed more discrepant sex differences in 2015-2017 alongside agomelatine and trazodone-all of which are ascribed a lower risk of weight gain while also having sedating and/ or sleep-promoting properties (Fachinfo-Service 2020). Perhaps this indicates that women were even less willing to tolerate potential weight gain during the later timeframe. This may not be without reason as women may be more susceptible to psychotropic drug-induced weight gain (Seeman 2009, 2020; Tandon et al. 2020) and have a harder time subsequently losing gained weight (Seeman 2009).

\section{Sex differences in biology and pharmacokinetics of psychotropic drugs}

Research suggests that sex strongly interacts with many brain function due to hormonal effects and effects of genomic sex (i.e., presence of two X-chromosomes or one X- and one Y-chromosome) (Rubinow and Schmidt 2019). These processes, though not understood in full, provide possible explanations for the higher incidence of MDD in females (Rubinow and Schmidt 2019) and are also postulated to have relevant effects on the treatment of depression (HernándezHernández et al. 2019). Progesterone, for example, has shown to induce an enhanced receptor binding of benzodiazepines making these drugs more potent when used in the treatment of women (Farkouh et al. 2020). This may present a possible explanation for the higher use of benzodiazepines in the treatment of women in this patient collective.

Furthermore, there are sex-related differences in the metabolism of drugs via cytochrome P450 (CYP) enzymes. CYP3A4 is well-researched on this behalf and has been found to be up to $50 \%$ more active in adult Caucasian women (Farkouh et al. 2020), while CYP1A2 activity is higher in males (Scandlyn et al. 2008). It seems rather unlikely that the consideration of CYP-enzyme profiles according to the patient's sex played a relevant role in a clinician's choice to prescribe a certain drug or refrain from doing so. However, activity of CYP-enzymes plays a decisive role in how a drug is metabolized and lastly how well it is tolerated by a patient. These implications may have led clinicians to observe that certain drugs are tolerated better when used in the treatment of either men or women. Under consideration of their pharmacokinetic profiles, these observations can be applied to some of the respective isoenzymes' substrates in this study. Trazodone, a substrate of CYP3A4 (Procyshyn et al. 2019), was used more in the treatment of female patients in this study, while the CYP1A2 substrate olanzapine (Procyshyn et al. 2019) showed higher use among males. This does not hold true for all sex differences observed among psychotropic drugs. For example, duloxetine is primarily metabolized via CYP1A2, while the major pharmacokinetic pathways of mirtazapine include CYP3A4 (Procyshyn et al. 2019). Consideration of activity of CYP-enzymes according to the above-mentioned sex differences alone will not guarantee a successful drug treatment-for this purpose, CYP testing to detect inter-individual variability in drug response remains the gold standard (Samer et al. 2013).

\section{Sex differences in help-seeking behavior, symptom presentation, and polypsychopharmacy}

Epidemiological studies on the prevalence of MDD consistently report that women have a two-fold increased risk of being diagnosed with MDD (Herzog et al. 2019). The higher willingness of women to seek professional help for their emotional problems is well-known (Frackiewicz et al. 2000). This of course does not apply in the same extent to the inpatients in this collective who are already in psychiatric treatment, but it may be one possible explanation for the higher use of psychotropic drugs among women in population-based settings. If men are more hesitant to report symptoms of MDD to a physician, concomitant psychotropic drug use arising from symptoms such as sleeping disorders would also be expected to be lower in the inpatient setting.

In general, women with MDD are three times more likely to present with atypical symptoms (e.g., anxiety, irritability, increased appetite) (Halbreich and Kahn 2007). Females are more likely to ruminate, complain of somatic discomfort, such as autonomic cardio-respiratory and gastrointestinal symptoms, and suffer from comorbid psychiatric diagnoses, such as anxiety, panic, and eating disorders (Gorman 2006; Halbreich and Kahn 2007). Somatic symptoms have shown to preferentially respond to SSRIs (LeGates et al. 2019), however, these manifestations may not be sufficiently alleviated by treatment with an ADD alone, therefore leading to the prescription of other psychotropic drugs. This may explain why female patients in this study, but also in other studies (Boyd et al. 2015), are more likely to be prescribed a higher number of psychotropic drugs, such as TRDs and HYPDs, which were both more frequently used in the treatment of women, as well as pregabalin - an AED primarily used for its anxiolytic and not its mood-stabilizing properties. Men, on the other hand, are more likely to externalize symptoms and report substance misuse, impulsivity, overinvolvement in work (Oliffe et al. 2019), and tension within interpersonal relationships (Altemus et al. 2014)—symptoms which are not included by diagnostic criteria resulting in frequent oversight (Oliffe et al. 2019). 
While female SSRI and NaSSA users were both more likely to simultaneously use HYPDs and TRDs, this study was unable to identify comparable sex differences among SSNRI users. This may indicate that SSNRIs cause more psychiatric ADRs, such as agitation, sleep disorders, and anxiety, in general which in turn raise the demand for symptom-driven prescription of sedating drugs, such as HYPDs and TRDs, regardless of sex. This study found that male venlafaxine users were more likely to concomitantly utilize other psychotropic drugs with sedating properties, such as mirtazapine and SGAs, than their female counterparts, perhaps again indicating that men are more willing to utilize weight gain-inducing drugs to achieve sedating effects.

Apart from the use of psychotropic drugs, the treatment of MDD includes numerous non-pharmacological treatment strategies, such as psychotherapy and electroconvulsive therapy (ECT). A minority (i.e., 1.72\%) of patients with affective disorders in Germany are prescribed ECT (Timäus et al. 2021). Females have been reported to more often receive ECT (Timäus et al. 2021; Buley et al. 2017; Wood and Burgess 2003), while at the same time, recipients of ECT are treated with a substantially higher number of ADDs, APDs, HYPDs, and TRDs (Wilkinson et al. 2018). Psychotherapy, on the other hand, is much more commonly encountered treatment method. Outpatient data from Germany suggest that women are more likely to receive psychotherapy (Epping et al. 2017). The combined use of psychotherapy and pharmacotherapy has been associated with superior outcomes in the treatment of MDD than either strategy alone (Cuijpers et al. 2014). While data on concurrent ECT and psychotherapy are not available for this patient collective, these findings from other studies may further implicate an increased willingness of females to seek and acquire mental health care both of pharmacological and non-pharmacological entity.

\section{Limitations}

The data presented here only represent a descriptive analysis of the current state of drug utilization based on sex during a 17-year time period. Other clinically relevant aspects also potentially significantly determining the selection of psychotropic drugs, such as patient age, (psychiatric) comorbidity, and severity of MDD, were not considered in this study. As collected data and reported diagnoses are based on routine clinical data, certain aspects (e.g., somatic diagnoses, other sociodemographic characteristics) were not fully documented. Information on the use of non-pharmacological treatment options, such as psychotherapy and ECT, is not accessible. Further, data regarding clinical aspects of treatment, such as course of clinical symptoms and the occurrence of ADRs, as well as (psychiatric) comorbidities indicating the use of a psychotropic drug (such as AEDs for epilepsy) was not available. Therefore, the rationale behind the selection of a certain drug/drug group was not verifiable for this collective of inpatients. It would also not be retraceable, if the utilization of multiple drugs was due to cross-tapering strategies to some extent when switching from one psychotropic drug to another, thus resulting in an overestimation of utilization rates and polypsychopharmacy.

\section{Conclusion}

This study examining the psychopharmacological treatment of inpatients with MDD detected several relevant differences between men and women from 2001 to 2017 . Monotherapy was more common among male patients, whereas women had a higher risk of being treated with four or more psychotropic drugs. Men and women were equally likely to be treated with ADDs. Utilization of NaSSAs (mainly mirtazapine) and bupropion was higher among men, whereas other ADD-subgroups (e.g., SSRIs and TCAs) showed only minimal sex differences. APDs were used in the treatment of almost half of both men and women, with an increasing utilization trend in 2015-2017, especially among men. Men had higher use of SGAs, whereas women were more likely to use lp FGAs. Use of TRDs and HYPDs showed a consistently higher utilization in female inpatients, which was even more pronounced for HYPD use among women in 2015-2017.

The clinical data presented here show that men and women with MDD are treated differently in terms of psychopharmacological approaches. While the sex differences found in this study are not as large as found by other authors among the ambulatory setting, they are clearly detectable, especially in the later timeframe of this study (i.e., 2015-2017). This indicates that the wide-spread discussion of the effect of sex is also finding consideration in the treatment of MDD in naturalistic, clinical settings. Current research suggests that sex differences in the development and treatment of MDD may be of unprecedented importance. Sex-specific recommendations in the treatment of MDD are yet to be made. Further research is needed to validate and better comprehend sex differences in the treatment response to psychotropic drugs.

Acknowledgements The authors would like to thank all psychiatric hospitals participating in the AMSP project for their continuous support in data collection.

Author contributions JS extensively revised the first draft of the manuscript, performed further statistical analysis, interpretation of the data, and research. FF wrote the first draft of the manuscript, performed initial statistical analysis, and interpretation of the data. XB performed initial statistical analysis and research. RE performed and conceptualized statistical analysis, interpretation of the data, and revised various versions of the manuscript. SB, SS, ER, and MR provided critical 
revision of the manuscript. ST supervised the project, interpreted the data, and performed various revisions of the manuscript. RG drew data from the AMSP data pool, conceptualized statistical analysis, interpreted the data, and performed various revisions of the manuscript. MS and WG conceptualized the project and provided supervision and critical revision of the manuscript. All authors contributed to and approved the final manuscript.

Funding Open Access funding enabled and organized by Projekt DEAL. The research presented in this manuscript did not receive any specific Grants or Funding. The AMSP drug safety project is facilitated by non-profit associations in Germany, Austria, and Switzerland. The AMSP project has been supported with unrestricted educational and research grants since 1993 by the following companies:

German companies: Abbott $\mathrm{GmbH} \& \mathrm{Co}$. KG, AstraZeneca GmbH, Aventis Pharma Deutschland GmbH GE-O/R/N, Bayer Vital $\mathrm{GmbH}$, Boehringer Mannheim GmbH, Bristol-Myers-Squibb, Ciba Geigy GmbH, Desitin Arzneimittel GmbH, Duphar Pharma GmbH \& Co. KG, Eisai GmbH, Esparma GmbH Arzneimittel, GlaxoSmithKline Pharma GmbH \& Co. KG, Hoffmann-La Roche AG Medical Affairs, Janssen-Cilag GmbH, Janssen Research Foundation, Knoll Deutschland GmbH, Lilly Deutschland GmbH Niederlassung Bad Homburg, Lundbeck GmbH \& Co. KG, Novartis Pharma GmbH, Nordmark Arzneimittel GmbH, Organon GmbH, Otsuka-Pharma Frankfurt, Pfizer GmbH, Pharmacia \& Upjohn GmbH, Promonta Lundbeck Arzneimittel, Recordati Pharma GmbH, Rhone-Poulenc Rohrer, Sanofi-Synthelabo GmbH, Sanofi-Aventis Deutschland, Schering AG, SmithKlineBeecham Pharma GmbH, Solvay Arzneimittel GmbH, Synthelabo Arzneimittel GmbH, Dr. Wilmar Schwabe GmbH \& Co., Thiemann Arzneimittel GmbH, Troponwerke GmbH \& Co. KG, Upjohn $\mathrm{GmbH}$, Wander Pharma GmbH, and Wyeth-Pharma GmbH.

Austrian companies: Astra Zeneca Österreich GmbH, Boehringer Ingelheim Austria, Bristol-Myers Squibb GmbH, CSC Pharmaceuticals $\mathrm{GmbH}$, Eli Lilly GmbH, Germania Pharma GmbH, GlaxoSmithKline Pharma GmbH, Janssen-Cilag Pharma GmbH, Lundbeck GmbH, Novartis Pharma GmbH, Pfizer Med Inform, and Wyeth Lederle Pharma GmbH.

Swiss companies: AHP (Schweiz) AG, AstraZeneca AG, BristolMyers Squibb AG, Desitin Pharma GmbH, Eli Lilly (Suisse) S.A., Essex Chemie AG, GlaxoSmithKline AG, Janssen-Cilag AG, Lundbeck (Suisse) AG, Organon AG, Pfizer AG, Pharmacia, Sanofi-Aventis (Suisse) S.A., Sanofi-Synthelabo SA, Servier SA, SmithKlineBeecham AG, Solvay Pharma AG, Wyeth AHP (Suisse) AG, and Wyeth Pharmaceuticals AG.

\section{Declarations}

Conflict of interest JS took part in an educational event sponsored by Otsuka/Lundbeck. ST is a member of the advisory board for Otsuka and Janssen-Cilag and has received speaker's honoraria from JanssenCilag, Lundbeck/Otsuka and Servier. All other authors state they have no conflicts of interest to declare.

Open Access This article is licensed under a Creative Commons Attribution 4.0 International License, which permits use, sharing, adaptation, distribution and reproduction in any medium or format, as long as you give appropriate credit to the original author(s) and the source, provide a link to the Creative Commons licence, and indicate if changes were made. The images or other third party material in this article are included in the article's Creative Commons licence, unless indicated otherwise in a credit line to the material. If material is not included in the article's Creative Commons licence and your intended use is not permitted by statutory regulation or exceeds the permitted use, you will need to obtain permission directly from the copyright holder. To view a copy of this licence, visit http://creativecommons.org/licenses/by/4.0/.

\section{References}

Altemus M, Sarvaiya N, Neill Epperson C (2014) Sex differences in anxiety and depression clinical perspectives. Front Neuroendocrinol 35(3):320-330. https://doi.org/10.1016/j.yfrne.2014.05.004

Boyd A, Van de Velde S, Pivette M, ten Have M, Florescu S, O’Neill S, Caldas-de-Almeida JM, Vilagut G, Haro JM, Alonso J, KovessMasféty V (2015) Gender differences in psychotropic use across Europe: results from a large cross-sectional, population-based study. Eur Psychiatry 30(6):778-788. https://doi.org/10.1016/j. eurpsy.2015.05.001

Buley N, Copland E, Hodge S, Chaplin R (2017) A further decrease in the rates of administration of electroconvulsive therapy in England. J Ect 33(3):198-202. https://doi.org/10.1097/yct.00000 00000000374

Cleare A, Pariante CM, Young AH, Anderson IM, Christmas D, Cowen PJ, Dickens C, Ferrier IN, Geddes J, Gilbody S, Haddad PM, Katona C, Lewis G, Malizia A, McAllister-Williams RH, Ramchandani P, Scott J, Taylor D, Uher R (2015) Evidence-based guidelines for treating depressive disorders with antidepressants: a revision of the 2008 British Association for Psychopharmacology guidelines. J Psychopharmacol 29(5):459-525. https://doi.org/10. 1177/0269881115581093

Cuijpers P, Sijbrandij M, Koole SL, Andersson G, Beekman AT, Reynolds CF 3rd (2014) Adding psychotherapy to antidepressant medication in depression and anxiety disorders: a meta-analysis. World Psychiatry 13(1):56-67. https://doi.org/10.1002/wps.20089

DGPPN, BÄK, KBV, AWMF (ed) (2017) für die Leitliniengruppe Unipolare Depression. S3-Leitlinie/Nationale VersorgungsLeitline Unipolare Depression-Langfassung, 2. Auflage. Version 5. 2015. https://doi.org/10.6101/AZQ/000364

Engel RR, Grohmann R, Rüther E, Hippius H (2004) Research methods in drug surveillance. Pharmacopsychiatry 37(Suppl 1):S12-15. https://doi.org/10.1055/s-2004-815506

Epping J, Muschik D, Geyer S (2017) Social inequalities in the utilization of outpatient psychotherapy: analyses of registry data from German statutory health insurance. Int J Equity Health 16(1):147. https://doi.org/10.1186/s12939-017-0644-5

Estancial Fernandes CS, de Azevedo RCS, Goldbaum M, Barros MBdA (2018) Psychotropic use patterns: are there differences between men and women? PLoS ONE 13(11):e0207921e0207921. https://doi.org/10.1371/journal.pone.0207921

Fachinfo-Service Fachinformationsverzeichnis Deutschland. www. fachinfo.de. Accessed 20 Apr 2020

Farkouh A, Riedl T, Gottardi R, Czejka M, Kautzky-Willer A (2020) Sex-related differences in pharmacokinetics and pharmacodynamics of frequently prescribed drugs: a review of the literature. Adv Ther 37(2):644-655. https://doi.org/10.1007/s12325-019-01201-3

Frackiewicz EJ, Sramek JJ, Cutler NR (2000) Gender differences in depression and antidepressant pharmacokinetics and adverse events. Ann Pharmacother 34(1):80-88. https://doi.org/10.1345/ aph. 18465

Franconi F, Campesi I (2014) Pharmacogenomics, pharmacokinetics and pharmacodynamics: interaction with biological differences between men and women. Br J Pharmacol 171(3):580-594. https://doi.org/10.1111/bph.12362

González-López MC, Rodríguez-López CM, Parrón-Carreño T, Luna JD, Del Pozo E (2015) Trends in the dispensation of antidepressant drugs over the past decade (2000-2010) in Andalusia. Spain 
Soc Psychiatry Psychiatr Epidemiol 50(5):705-712. https://doi. org/10.1007/s00127-014-0995-9

Gorman JM (2006) Gender differences in depression and response to psychotropic medication. Gend Med 3(2):93-109. https://doi.org/ 10.1016/s1550-8579(06)80199-3

Gotlib D, Ramaswamy R, Kurlander JE, DeRiggi A, Riba M (2017) Valproic acid in women and girls of childbearing age. Curr Psychiatry Rep 19(9):58. https://doi.org/10.1007/s11920-017-0809-3

Grohmann R, Engel RR, Ruether E, Hippius H (2004) The AMSP drug safety program: methods and global results. Pharmacopsychiatry 37:S4-S11

Grohmann R, Engel RR, Moller HJ, Ruther E, van der Velden JW, Stubner S (2014) Flupentixol use and adverse reactions in comparison with other common first- and second-generation antipsychotics: data from the AMSP study. Eur Arch Psychiatry Clin Neurosci 264(2):131-141. https://doi.org/10.1007/s00406-013-0419-y

Halbreich U, Kahn LS (2007) Atypical depression, somatic depression and anxious depression in women: are they gender-preferred phenotypes? J Affect Disord 102(1-3):245-258. https://doi.org/ 10.1016/j.jad.2006.09.023

Hernández-Hernández OT, Martínez-Mota L, Herrera-Pérez JJ, JiménezRubio G (2019) Role of estradiol in the expression of genes involved in serotonin neurotransmission: implications for female depression. Curr Neuropharmacol 17(5):459-471. https://doi.org/10.2174/ 1570159x16666180628165107

Herzog DP, Wegener G, Lieb K, Müller MB, Treccani G (2019) Decoding the mechanism of action of rapid-acting antidepressant treatment strategies: does gender matter? Int J Mol Sci. https://doi.org/ 10.3390/ijms20040949

Karanti A, Bobeck C, Osterman M, Kardell M, Tidemalm D, Runeson B, Lichtenstein P, Landén M (2015) Gender differences in the treatment of patients with bipolar disorder: a study of 7354 patients. J Affect Disord 174:303-309. https://doi.org/10.1016/j.jad.2014.11.058

Khan A, Brodhead AE, Schwartz KA, Kolts RL, Brown WA (2005) Sex differences in antidepressant response in recent antidepressant clinical trials. J Clin Psychopharmacol 25(4):318-324. https://doi.org/ 10.1097/01.jcp.0000168879.03169.ce

LeGates TA, Kvarta MD, Thompson SM (2019) Sex differences in antidepressant efficacy. Neuropsychopharmacology 44(1):140-154. https://doi.org/10.1038/s41386-018-0156-z

Liu KA, Mager NA (2016) Women's involvement in clinical trials: historical perspective and future implications. Pharm Pract (granada) 14(1):708. https://doi.org/10.18549/PharmPract.2016.01.708

Liu Q, He H, Yang J, Feng X, Zhao F, Lyu J (2019) Changes in the global burden of depression from 1990 to 2017: findings from the Global Burden of Disease study. J Psychiatr Res. https://doi.org/10.1016/j. jpsychires.2019.08.002

Luo Y, Kataoka Y, Ostinelli EG, Cipriani A, Furukawa TA (2020) National prescription patterns of antidepressants in the treatment of adults with major depression in the us between 1996 and 2015: a population representative survey based analysis. Front Psychiatry 11:35. https://doi.org/10.3389/fpsyt.2020.00035

Montejo AL, Prieto N, de Alarcón R, Casado-Espada N, de la Iglesia J, Montejo L (2019b) management strategies for antidepressant-related sexual dysfunction: a clinical approach. J Clin Med. https://doi.org/ $10.3390 / \mathrm{jcm} 8101640$

Montejo AL, Calama J, Rico-Villademoros F, Montejo L, GonzálezGarcía N, Pérez J (2019a) A real-world study on antidepressantassociated sexual dysfunction in 2144 outpatients: the SALSEX I Study. Arch Sex Behav 48(3):923-933. https://doi.org/10.1007/ s10508-018-1365-6

Oliffe JL, Rossnagel E, Seidler ZE, Kealy D, Ogrodniczuk JS, Rice SM (2019) Men's depression and suicide. Curr Psychiatry Rep 21(10):103. https://doi.org/10.1007/s11920-019-1088-y

Procyshyn RM, Bezchilbynk-Butler KZ, Jeffries JJ (2019) Clinical handbook of psychotropic drugs, vol 23. Hogrefe Publishing, USA
Rubinow DR, Schmidt PJ (2019) Sex differences and the neurobiology of affective disorders. Neuropsychopharmacology 44(1):111-128. https://doi.org/10.1038/s41386-018-0148-z

Samer CF, Lorenzini KI, Rollason V, Daali Y, Desmeules JA (2013) Applications of CYP450 testing in the clinical setting. Mol Diagn Ther 17(3):165-184. https://doi.org/10.1007/s40291-013-0028-5

Sansone RA, Sansone LA (2012) Antidepressant adherence: are patients taking their medications? Innov Clin Neurosci 9(5-6):41-46

Scandlyn MJ, Stuart EC, Rosengren RJ (2008) Sex-specific differences in CYP450 isoforms in humans. Expert Opin Drug Metab Toxicol 4(4):413-424. https://doi.org/10.1517/17425255.4.4.413

Schneider M, Pauwels P, Toto S, Bleich S, Grohmann R, Heinze M, Greiner T (2020) Severe weight gain as an adverse drug reaction of psychotropics: data from the AMSP project between 2001 and 2016. Eur Neuropsychopharmacol 36:60-71. https://doi.org/10. 1016/j.euroneuro.2020.05.001

Seeman MV (2009) Secondary effects of antipsychotics: women at greater risk than men. Schizophr Bull 35(5):937-948. https://doi. org $/ 10.1093 / \mathrm{schbul} / \mathrm{sbn} 023$

Seeman MV (2020) Men and women respond differently to antipsychotic drugs. Neuropharmacology. https://doi.org/10.1016/j.neuropharm. 2019.05.008

Seifert J, Engel RR, Bernegger X, Führmann F, Bleich S, Stübner S, Sieberer M, Greil W, Toto S, Grohmann R (2021) Time trends in pharmacological treatment of major depressive disorder: results from the AMSP pharmacovigilance program from 2001 to 2017. J Affect Disord 281:547-556. https://doi.org/10.1016/j.jad.2020.12.073

Serna C, Cruz I, Galván L, Real J, Gascó E, Soler-González J (2010) Evolution of the prevalence and incidence of consumption of antidepressants in a Spanish region (2002-2007). Ment Health Fam Med 7(1):9-15

Sramek JJ, Murphy MF, Cutler NR (2016) Sex differences in the psychopharmacological treatment of depression. Dialogues Clin Neurosci 18(4):447-457

Subramaniam M, He VYF, Vaingankar JA, Abdin E, Chong SA (2013) Prevalence of and factors related to the use of antidepressants and benzodiazepines: results from the Singapore Mental Health Study. BMC Psychiatry 13(1):231. https://doi.org/10.1186/ 1471-244X-13-231

Sundell KA, Gissler M, Petzold M, Waern M (2011) Antidepressant utilization patterns and mortality in Swedish men and women aged 20-34 years. Eur J Clin Pharmacol 67(2):169-178. https://doi.org/ 10.1007/s00228-010-0933-z

Tandon R, Lenderking WR, Weiss C, Shalhoub H, Barbosa CD, Chen J, Greene M, Meehan SR, Duvold LB, Arango C, Agid O, Castle D (2020) The impact on functioning of second-generation antipsychotic medication side effects for patients with schizophrenia: a worldwide, cross-sectional, web-based survey. Ann Gen Psychiatry 19:42. https://doi.org/10.1186/s12991-020-00292-5

The National Institute for Health Care Excellence (2009) Depression in adults: recognition and mangement (CG90). Clinical guideline. www.nice.org.uk/guidance/cg90

Timäus C, Vogelgsang J, Kis B, Radenbach K, Wolff-Menzler C, Mavridou K, Gyßer S, Hessmann P, Wiltfang J (2021) Current clinical practice of electroconvulsive therapy and repetitive transcranial magnetic stimulation in psychiatry, a German sample. Eur Arch Psychiatry Clin Neurosci 271(1):181-190. https://doi.org/10.1007/ s00406-020-01099-X

Van der Heyden JHA, Gisle L, Hesse E, Demarest S, Drieskens S, Tafforeau J (2009) Gender differences in the use of anxiolytics and antidepressants: a population based study. Pharmacoepidemiol Drug Saf 18(11):1101-1110. https://doi.org/10.1002/pds.1827

Wilkinson ST, Agbese E, Leslie DL, Rosenheck RA (2018) Identifying recipients of electroconvulsive therapy: data from privately insured americans. Psychiatr Serv 69(5):542-548. https://doi.org/10.1176/ appi.ps.201700364 
Wood DA, Burgess PM (2003) Epidemiological analysis of electroconvulsive therapy in Victoria Australia. Aust N Z J Psychiatry 37(3):307-311. https://doi.org/10.1046/j.1440-1614.2003.01182.x

World Health Organization (1992) The ICD-10 classification of mental and behavioural disorders: clinical descriptions and diagnostic guidelines. World Health Organization (WHO), Geneva

Yu Z, Zhang J, Zheng Y, Yu L (2020) Trends in antidepressant use and expenditure in six major cities in China from 2013 to 2018. Front Psychiatry. https://doi.org/10.3389/fpsyt.2020.00551

Zhong W, Kremers HM, Yawn BP, Bobo WV, St Sauver JL, Ebbert JO, Finney Rutten LJ, Jacobson DJ, Brue SM, Rocca WA (2014) Time trends of antidepressant drug prescriptions in men versus women in a geographically defined US population. Arch Womens Ment Health 17(6):485-492. https://doi.org/10.1007/s00737-014-0450-7

Publisher's Note Springer Nature remains neutral with regard to jurisdictional claims in published maps and institutional affiliations. 